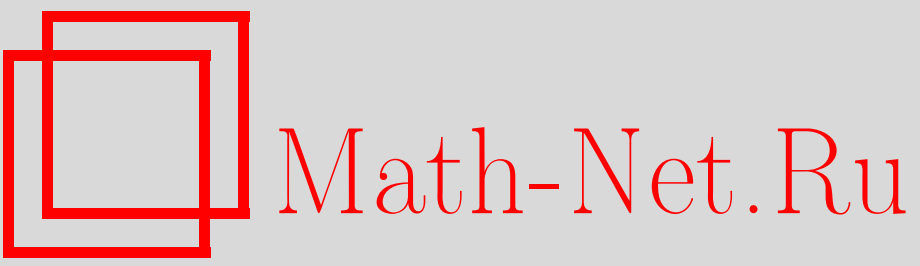

М. В. Маханова, В. Б. Приезжев, Нестационарные вероятности и временные корреляционные функции для асимметричного процесса с исключенным объемом, ТMФ, 2006, том 146, номер 3, 499-508

DOI: https://doi.org/10.4213/tmf2050

Использование Общероссийского математического портала Math-Net.Ru подразумевает, что вы прочитали и согласны с пользовательским соглашением

http: //www.mathnet.ru/rus/agreement

Параметры загрузки:

IP: 52.6 .47 .48

26 апреля 2023 г., 06:08:51 
ТЕОРЕТИЧЕСКАЯ

И МАТЕМАТИЧЕСКАЯ

ФИЗИКА

Том 146, № 3

март, 2006

(C) 2006 г.

М. В. Маханова*, В. Б. Приезжев*

\section{НЕСТАЦИОНАРНЫЕ ВЕРОЯТНОСТИ И ВРЕМЕННЫ์ЫЕ КОРРЕЛЯЦИОННЫЕ ФУНКЦИИ ДЛЯ АСИММЕТРИЧНОГО ПРОЦЕССА С ИСКЛЮЧЕННЫМ ОБЪЕМОМ}

\footnotetext{
Представлено полное решение основного кинетического уравнения системы взаимодействующих частиц с конечной плотностью. Решение получено с помощью новой формы подстановки Бете для простого асимметричного процесса с исключенным объемом на кольце. Для дискретного случая процесса впервые найдена одноточечная временна́я корреляционная функция.
}

Ключевые слова: одномерные модели взаимодействующих частиц, подстановка Бете, условная вероятность, корреляционная функция.

\section{1. ВВЕДЕНИЕ}

В неравновесной статистической механике состояние системы взаимодействующих частиц дается решением основного кинетического уравнения

$$
\frac{d P(\alpha ; t)}{d t}=\sum_{\gamma}\left[W_{\gamma \alpha} P(\gamma ; t)-W_{\alpha \gamma} P(\alpha ; t)\right],
$$

где $\alpha, \gamma$ - состояния системы, $W_{\alpha \gamma}$ - скорость перехода из состояния $\alpha$ в состояние $\gamma$, $P(\alpha ; t)$ - вероятность того, что система находится в состоянии $\alpha$ в момент времени $t$ при условии, что известно состояние $\alpha_{0}$ системы в момент времени $t=0$. Из-за экспоненциального роста числа состояний с увеличением размера системы эта задача никогда не была решена полностью не только для реальных систем, но даже для упрощенных одномерных моделей взаимодействующих частиц в конечном объеме.

Среди простых одномерных моделей наиболее перспективным кандидатом для точного решения уравнения (1) является простой асимметричный процесс с исключенным объемом (ASEP) [1], [2]. В течение последнего десятилетия было точно вычислено множество асимптотических свойств ASEP в пределе больших интервалов времени (см., например, [3]-[6]) и получено нестационарное решение уравнения (1)

*Объединенный институт ядерных исследований, Дубна, Московская обл., Россия. E-mail: mvmakhan@thsun1.jinr.ru, priezzvb@thsun1.jinr.ru 
для конечного числа частиц на бесконечной решетке [7]. Очевидным недостатком такого решения является то, что в асимптотическом пределе плотность частиц стремится к нулю, а динамика приближается к тривиальному случаю невзаимодействующих частиц. Поэтому остается актуальной проблема решения уравнения (1) для систем с конечной плотностью частиц. Первая цель этой статьи - представить полное решение уравнения (1) для ASEP на кольце, содержащем $L$ узлов и $P$ частиц. Вторая цель - найти простейшую временну́ю корреляционную функцию для дискретного процесса.

Для непрерывного времени ASEP описывается уравнением (1), где скорости переходов определяются согласно правилу исключения: в течение бесконечно малого интервала времени $d t$ каждая частица с вероятностью $d t$ совершает прыжок на один узел вправо, если он свободен. Если этот узел занят, то движение запрещено. Кроме непрерывной формулировки процесса существует также дискретная, которая удобна для иллюстрации и численных расчетов.

Введем квадратную решетку $\Lambda$ и обозначим положение частицы целочисленными координатами $(x, t)$. Вертикальная ось времени направлена вниз, горизонтальная пространственная ось направлена вправо. Мы рассматриваем движение частиц на кольце, а их траектории на периодической решетке $\Lambda$, с периодом, равным размеру кольца. Траектория каждой частицы представляет собой последовательность единичных вертикальных и диагональных отрезков, соединяющих узлы решетки. Каждый диагональный отрезок соответствует прыжку частицы вправо за единицу времени и имеет вес $z$. Вертикальный отрезок имеет вес $y$ и соответствует тому, что частица за единицу времени не изменила своего положения.

Дискретный ASEP генерирует траектории $P$ частиц, начинающиеся в точках кольца $0 \leqslant x_{1}^{0}<x_{2}^{0}<\cdots<x_{P}^{0}<L$ и заканчивающиеся в точках $0 \leqslant x_{1}<x_{2}<\cdots<$ $x_{P}<L$ после произвольного числа обходов вокруг кольца в течение времени $t$. Согласно правилу исключения траектории частиц не пересекаются, а в случае, когда два вертикальных единичных отрезка расположены в соседних узлах, вес левого изменяется с $y$ на 1 (частица с вероятностью 1 остается на месте, пока соседний справа узел занят).

Производящая функция всех траекторий свободно движущейся частицы за время $t$, с весом каждого шага $z$ или $y$, есть полином $(z+y)^{t}$, а для системы из $P$ свободных частиц - соответственно $(z+y)^{t P}$. Если производящая функция взаимодействующих частиц $G_{P}$ будет учитывать только те траектории, которые удовлетворяют начальным и конечным условиям, а также правилу исключения ASEP, то при выполнении равенства $z+y=1(z<1)$ функция $G_{P}$ есть условная вероятность $P\left(x_{1}, \ldots, x_{P} ; t \mid x_{1}^{0}, \ldots, x_{P}^{0} ; 0\right)$, причем $z$ - вероятность скачка частицы на свободный узел, а $y$ - вероятность остаться на месте, если соседний узел справа свободен.

Напишем для примера полином $G_{P}$ для случая движения двух частиц $(P=2)$ в течение времени $t=6$ по простейшему кольцу из трех звеньев, $L=3$. Начальные и конечные координаты равны соответственно $x_{1}^{0}=1, x_{2}^{0}=2$ и $x_{1}=1, x_{2}=2$. Если бы частицы двигались по бесконечной цепочке $(L \rightarrow \infty)$, то они имели бы лишь одну возможность попасть из $\left(x_{1}^{0}, x_{2}^{0}\right)$ в $\left(x_{1}, x_{2}\right)$ - оставаться на месте в тече- 


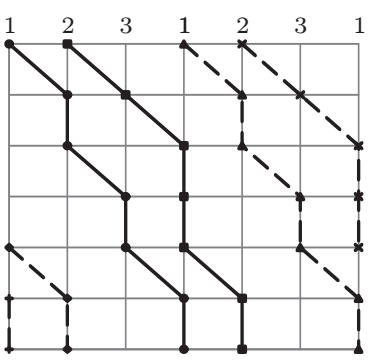

$z^{6} y^{4}$

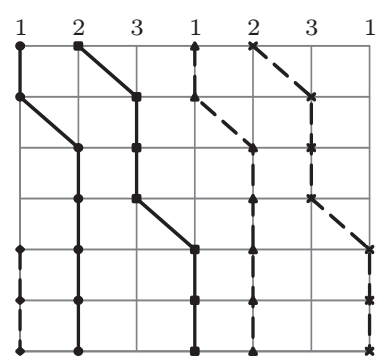

$z^{3} y^{6}$

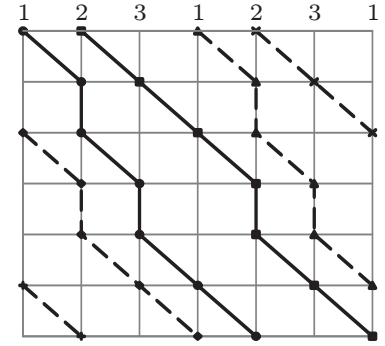

$z^{9} y^{2}$

Рис. 1. Траектории частиц на периодической решетке: $L=3, P=2$, $t=6$. Под каждым примером приведено слагаемое, которое данный набор траекторий вносит в величину $G_{2}$.

ние всего времени $t$. В этом случае $G_{2}=P(1,2 ; 0 \mid 1,2 ; 6)=y^{6}$. Периодичность в пространственном направлении решетки дает нам 243 способа прийти из заданных начальных точек в конечные: $G_{2}=z^{12}+30 z^{9} y^{2}+20 z^{9} y^{3}+z^{6} y^{6}+30 z^{6} y^{5}+90 z^{6} y^{4}+$ $20 z^{6} y^{3}+20 z^{3} y^{6}+30 z^{3} y^{5}+y^{6}$. Несколько примеров возможных конфигураций траекторий представлены на рис. 1.

Главным результатом, представленным в статье, является полное решение уравнения (1) для асимметричного процесса с исключениями на кольце, содержащем $L$ узлов и $P$ частиц. В разделе 2 сформулирован обобщенный анзац Бете, учитывающий периодические граничные условия, и получено точное выражение для условной вероятности $P\left(x_{1}, \ldots, x_{P} ; t \mid x_{1}^{0}, \ldots, x_{P}^{0} ; 0\right)$ обнаружить частицы в заданных точках $\left(x_{1}, \ldots, x_{P}\right)$ после эволюции в течение времени $t$ из начального состояния $\left(x_{1}^{0}, \ldots, x_{P}^{0}\right)$. В разделе 3 приведен расчет для временно́й одноточечной корреляционной функции дискретного процесса.

\section{2. ТОЧНОЕ НЕСТАЦИОНАРНОЕ РЕШЕНИЕ ДЛЯ УСЛОВНОЙ ВЕРОЯТНОСТИ}

Обозначим через $B(N, t)$ вероятность для свободной частицы прийти из точки $\left(x^{0}, 0\right)$ в точку $(x, t)$ за $t$ дискретных шагов: $B(N, t)=\left(\begin{array}{l}t \\ N\end{array}\right) z^{N} y^{t-N}$, где $N=x-x^{0}$ - расстояние, пройденное частицей. В непрерывном пределе $z \rightarrow 0, t \rightarrow \infty$ мы имеем $B(N, t)=e^{-t} t^{N} / N$ !, где $t-$ непрерывное время, перемасштабированное так, что $t z \rightarrow t$. Для $N<0$ мы принимаем $B(N, t)=0$.

Следуя [7], введем функцию $F_{m}$ для любого целого $m$ такую, что

$$
F_{m}\left(x_{i}^{0}, x_{j} \mid t\right)=\sum_{k=0}^{\infty}\left(\begin{array}{c}
k+m-1 \\
m-1
\end{array}\right) F_{0}\left(x_{i}^{0}-k, x_{j} \mid t\right),
$$

если $m>0$,

$$
F_{m}\left(x_{i}^{0}, x_{j} \mid t\right)=\sum_{k=0}^{-m}(-1)^{k}\left(\begin{array}{c}
-m \\
k
\end{array}\right) F_{0}\left(x_{i}^{0}-k, x_{j} \mid t\right),
$$


если $m<0$, и

$$
F_{0}\left(x_{i}^{0}, x_{j} \mid t\right)=B\left(x_{j}-x_{i}^{0}, t\right)
$$

если $m=0$.

Определим матрицу $M$ размером $P \times P$, элементы которой задаются функцией $F_{m}:$

$$
M_{i j}=F_{s_{i j}}\left(x_{i}^{0}, x_{j}+n_{j} L \mid t\right),
$$

где

$$
s_{i j}=(P-1) n_{j}-\sum_{k \neq j} n_{k}+j-i .
$$

Тогда справедлива следующая теорема [8].

Теорема. Для любых челых чисел $L, P$ maких, что $L>1,0<P<L$, производящая функиия $G_{P}$ есть

$$
G_{P}=\sum_{n_{1}=-\infty}^{\infty} \cdots \sum_{n_{P}=-\infty}^{\infty}(-1)^{\sum_{i<j}\left|n_{i}-n_{j}\right|} \operatorname{det} M .
$$

Получение этого результата основано на новой форме анзаца Бете, которая позволяет нам учитывать геометрические свойства траекторий частиц, движущихся по кольцу. Общим свойством интегрируемых моделей, допускающих двумерное пространственно-временное представление, является неизбежное пересечение двух траекторий при перестановке их конечных точек. Идея анзаца Бете состоит в том, чтобы представить взаимодействующие траектории частиц как набор свободных траекторий. Далее, используя однозначное соответствие между пересечениями траекторий и перестановками их концов, мы можем свести проблему перечисления всех взаимодействующих траекторий к правильному выбору знаков перестановок.

Начнем с двухчастичного случая, $P=2$. Удобно ввести на периодической оси $x$ новые координаты $X_{i}=x_{i}+n_{i} L$, где $n_{i}$ - целое число, учитывающее количество обходов по кольцу, $x_{i}-$ координата на кольце, $0 \leqslant x_{i}<L$. Вероятность $P\left(\alpha ; t \mid \alpha_{0} ; 0\right)$ того, что за время $t$ система перейдет из состояния $\alpha_{0}$ в состояние $\alpha$, можно написать в виде

$$
P\left(x_{1}, x_{2} ; t \mid x_{1}^{0}, x_{2}^{0} ; 0\right)=\sum_{X_{1}, X_{2}} \psi\left(X_{1}, X_{2} ; t\right),
$$

где суммирование происходит по всем $X_{1}, X_{2}$, которые удовлетворяют условиям $X_{1}=x_{1}+n_{1}^{*} L, X_{2}=x_{2}+n_{2}^{*} L$ или $X_{1}=x_{2}+n_{2}^{*} L, X_{2}=x_{1}+n_{1}^{*} L$, где $n_{1}^{*}, n_{2}^{*}$ - целые числа. Функция $\psi\left(X_{1}, X_{2} ; t\right)$ есть вероятность того, что первая частица придет в точку $X_{1}$, выйдя из начальной точки $x_{1}^{0}$, а вторая частица придет в $X_{2}$ из $x_{2}^{0}$, при условии, что траектории частиц подчиняются правилам ASEP.

Так же как $P\left(x_{1}, x_{2} ; t\right)$, функция $\psi\left(X_{1}, X_{2} ; t\right)$ удовлетворяет основному уравнению $(1)$, которое для двух частиц записывается в виде

$$
\frac{d \psi}{d t}=\psi\left(X_{1}-1, X_{2} ; t\right)+\psi\left(X_{1}, X_{2}-1 ; t\right)-2 \psi\left(X_{1}, X_{2} ; t\right) .
$$


Это уравнение необходимо дополнить граничными условиями [8]

$$
\begin{aligned}
\psi(X, X ; t) & =\psi(X, X+1 ; t), \\
\psi(X, X+L ; t) & =\psi(X+1, X+L ; t) \quad \forall X, t \geqslant 0 .
\end{aligned}
$$

Первое из них ставит запрет на ситуацию, когда первая частица обгоняет вторую (при этом частицы в некоторый момент времени оказались бы в одном узле решетки, что невозможно), второе запрещает второй частице, сделав дополнительный оборот по кольцу, обгонять первую.

Прежде всего перейдем к задаче о собственных значениях с помощью подстановки $\psi\left(X_{1}, X_{2} ; t\right)=e^{\lambda t} \psi\left(X_{1}, X_{2}\right)$ (по аналогии с $\left.P\left(x_{1}, x_{2} ; t\right)=e^{\lambda t} P\left(x_{1}, x_{2}\right)\right)$, тогда уравнение (9) запишется в виде

$$
\lambda \psi=\psi\left(X_{1}-1, X_{2}\right)+\psi\left(X_{1}, X_{2}-1\right)-2 \psi\left(X_{1}, X_{2}\right),
$$

с граничными условиями

$$
\begin{aligned}
\psi(X, X) & =\psi(X, X+1), \\
\psi(X, X+L) & =\psi(X+1, X+L) \quad \forall X \geqslant 0 .
\end{aligned}
$$

По методу Бете для нахождения собственной функции используется подстановка

$$
P\left(x_{1}, x_{2}\right)=A_{12} z_{1}^{x_{1}} z_{2}^{x_{2}}+A_{21} z_{1}^{x_{2}} z_{2}^{x_{1}},
$$

где параметры $z_{i}$ и $A_{i j}$ неизвестны. Они определяются из граничных и начальных условий. Вместо этой стандартной формы мы используем модифицированную подстановку:

$$
\psi\left(X_{1}, X_{2}\right)=\sum_{n_{1}, n_{2}}\left(A_{12}^{n_{1}, n_{2}} z_{1}^{x_{1}+n_{1} L} z_{2}^{x_{2}+n_{2} L}+A_{21}^{n_{2}, n_{1}} z_{1}^{x_{2}+n_{2} L} z_{2}^{x_{1}+n_{1} L}\right),
$$

где суммирование происходит по всем $n_{1}, n_{2}$, удовлетворяющим правилу $n_{1}+n_{2}=$ $n_{1}^{*}+n_{2}^{*}$, и параметры $A_{i j}$ зависят от порядка расположения траекторий.

Из уравнения (11) мы получаем $\lambda=-2+z_{1}^{-1}+z_{2}^{-1}$. Условия (12) будут удовлетворены, если

$$
\frac{A_{12}^{\left(n_{1}, n_{2}\right)}}{A_{21}^{\left(n_{1}, n_{2}\right)}}=-\frac{1-z_{1}}{1-z_{2}}
$$

и

$$
\frac{A_{12}^{\left(n_{1}, n_{2}\right)}}{A_{21}^{\left(n_{1}-1, n_{2}+1\right)}}=-\frac{1-z_{2}}{1-z_{1}} .
$$

Тогда параметры $A_{i j}^{\left(n_{1}, n_{2}\right)}$ можно найти с помощью рекурсии:

$$
A_{i j}^{\left(n_{1}, n_{2}\right)}=\operatorname{sign}(\pi)(-1)^{\left|n_{1}-n_{2}\right|} \frac{f\left(z_{1}, z_{2}\right)}{\left(1-z_{1}\right)^{s_{1 j}}\left(1-z_{2}\right)^{s_{2 j}}},
$$

где $s_{i j}$ определено соотношением (6), $f\left(z_{1}, z_{2}\right)$ - неизвестная функция и знак перестановки $\operatorname{sign}(\pi)$ положителен для $\{i, j\}=\{1,2\}$ и отрицателен для $\{i, j\}=\{2,1\}$. 
Используя обозначение $z_{j}=e^{i p_{j}}$, мы можем написать общее решение

$$
\psi\left(X_{1}, X_{2} ; t\right)=\int_{0}^{2 \pi} d p_{1} \int_{0}^{2 \pi} d p_{2} e^{\lambda t} \psi\left(X_{1}, X_{2}\right)
$$

и определить функцию $f\left(z_{1}, z_{2}\right)$ из начальных условий. В случае бесконечной решетки выбор

$$
f\left(z_{1}, z_{2}\right)=e^{-i p_{1} x_{1}^{0}-i p_{2} x_{2}^{0}}
$$

и определение полюсов в выражении (17) как $p_{j} \rightarrow p_{j}+i 0$ дает нам верные начальные условия

$$
P\left(x_{1}, x_{2} ; 0 \mid x_{1}^{0}, x_{2}^{0} ; 0\right)=\delta_{x_{1}, x_{1}^{0}} \delta_{x_{2}, x_{2}^{0}} .
$$

Произведя интегрирование в формуле (18), получаем

$$
\psi\left(X_{1}, X_{2} ; t\right)=\sum_{n_{1}+n_{2}=n_{1}^{*}+n_{2}^{*}}(-1)^{\left|n_{1}-n_{2}\right|} \operatorname{det} M,
$$

где $M$ - матрица размера $2 \times 2$, элементы которой определяются функцией (5). Суммирование по всем $n_{1}^{*}, n_{2}^{*}$ приводит к выражению (7) для $P=2$.

Для $P$ частиц вероятность $P(\alpha ; t \mid \alpha ; 0)$ принимает вид

$$
P\left(x_{1}, \ldots, x_{P} ; t\right)=\sum_{\{X\}} \psi\left(X_{1}, \ldots, X_{P} ; t\right)
$$

где все $X_{i}$ такие, что $X_{i}=x_{\pi(i)}+n_{\pi(i)}^{*}$, а $\pi$ - перестановки чисел $1,2, \ldots, P$. Функция $\psi\left(X_{1}, \ldots, X_{P} ; t\right)$ есть вероятность того, что $i$-я частица достигнет точки $X_{i}$, выйдя из точки $x_{i}^{0}, i=1,2, \ldots, P$, при условии, что траектории частиц удовлетворяют правилам ASEP. Мы снова используем подстановку $\psi\left(X_{1}, \ldots, X_{P} ; t\right)=e^{\lambda t} \psi\left(X_{1}, \ldots, X_{P}\right)$. Задачу на собственные значения для $P$ частиц мы получаем прямым обобщением (9), и ее необходимо дополнить граничными условиями, соответствующими $P$ возможным обгонам:

$$
\begin{aligned}
\psi\left(\ldots, X_{i}, X_{i}, \ldots\right) & =\psi\left(\ldots, X_{i}, X_{i}+1, \ldots\right), \\
\psi\left(X_{1}, X_{2}, \ldots, X_{1}+L\right) & =\psi\left(X_{1}+1, X_{2}, \ldots, X_{1}+L\right) .
\end{aligned}
$$

Анзац Бете принимает форму

$$
\psi\left(X_{1}, \ldots, X_{P}\right)=\sum_{\pi} \sum_{\{n\}} A_{\pi(1) \ldots \pi(P)}^{\left(n_{\pi(1)}, \ldots, n_{\pi(P)}\right)} \times \prod_{j=1}^{P} z_{j}^{x_{\pi(j)}+n_{\pi(j)} L},
$$

где первое суммирование производится по всем перестановкам $\pi$, а во втором выполняется правило $n_{1}+\cdots+n_{P}=n_{1}^{*}+\cdots+n_{P}^{*}$. Собственное значение для $P$ частиц равно

$$
\lambda=-P+\sum_{i=1}^{P} \frac{1}{z_{i}} .
$$

Чтобы найти параметры $A$ из граничных условий $(23)$, полезно ввести геометрическое объяснение. Заметим, что две пересекающиеся траектории неэквивалентны: 
одна из них принадлежит обгоняющей частице, и мы можем назвать ее активной, а вторая, наоборот, может быть названа пассивной. В случае бесконечной решетки активная и пассивная траектории расположены по порядку: для каждой пары частиц траектория $i$-й частицы по отношению к $(i+1)$-й частице всегда активна. На кольце любая из двух траекторий может быть активной или пассивной независимо от начальных условий. Более того, одна траектория может пересекать другую $m$ раз, если количество оборотов двух частиц отличается на $m$.

Из условий (23) следует, что

$$
\frac{A_{\ldots i j \ldots}^{\left(\ldots, n_{i}, n_{j}, \ldots\right)}}{A_{\ldots j i \ldots}^{\left(\ldots, n_{i}, n_{j}, \ldots\right)}}=-\frac{1-z_{i}}{1-z_{j}}
$$

$$
\frac{A_{\pi(1) \ldots \pi(P)}^{\left(n_{\pi(1)}, \ldots, n_{\pi(P)}\right)}}{A_{\pi(P) \ldots \pi(1)}^{\left(n_{\pi(1)}-1, \ldots, n_{\pi(P)}+1\right)}}=-\frac{1-z_{\pi(P)}}{1-z_{\pi(1)}}
$$

Отношения параметров $A$ в (26), (27) подобны отношениям $(15),(16)$, и их можно рассматривать как коэффициенты рассеяния, соответствующие пересечению двух частиц, что приводит к перестановке их конечных точек. Траектории частиц $i$ и $\pi(P)$ в выражениях (26), (27) активные, а траектории $j$ и $\pi(1)$ пассивные. Можно заключить, что каждое пересечение активной траектории $i$ добавляет множитель $\left(1-z_{i}\right)^{-1}$ в параметр $A_{\ldots j i \ldots}^{\left(\ldots, n_{i}, n_{j}, \ldots\right)}$, тогда как каждое пересечение пассивной траектории $j$ дает множитель $1-z_{j}$. Таким образом, показатель степени множителя $1-z_{i}$ для частицы, выходящей из $x_{i}^{0}$ и приходящей в $X_{j}$, определяется разностью между количеством активных и пассивных пересечений. Суммируя все вышесказанное, получим

$$
A_{\pi(1) \ldots \pi(P)}^{\left(n_{\pi(1)}, \ldots, n_{\pi(P)}\right)}=\frac{\operatorname{sign}(\pi)(-1)^{\sum_{i<j}\left|n_{i}-n_{j}\right|} f\left(z_{1}, \ldots, z_{P}\right)}{\left(1-z_{1}\right)^{s_{1 \pi(1)}} \ldots\left(1-z_{P}\right)^{s_{P \pi(P)}}},
$$

где знак правой части определяется количеством перестановок, а $f\left(z_{1}, \ldots, z_{P}\right)$ находим из начальных условий.

Условия (26), (27) принимают во внимание парное взаимодействие между частицами, когда две частицы находятся в соседних узлах кольца. Конфигурации, содержащие "плотно упакованные" интервалы из трех и более узлов, не дают новых ограничений, потому что граничные условия для любой пары не зависят от координат остальных частиц.

Как было указано выше, выбор

$$
f\left(z_{1}, \ldots, z_{p}\right)=\prod_{i=1}^{P} z_{i}^{-x_{i}^{0}}
$$

гарантирует выполнение начальных условий

$$
P\left(x_{1}, \ldots, x_{P} ; 0 \mid x_{1}^{0}, \ldots, x_{P}^{0} ; 0\right)=\delta_{x_{1}, x_{1}^{0}} \ldots \delta_{x_{P}, x_{P}^{0}} .
$$


Интегрируя $e^{\lambda t} \psi\left(X_{1}, \ldots, X_{P}\right)$ по $p_{j}=-i \ln z_{j}$ с параметрами $A$, которые даются соотношением (28), мы получаем функцию $\psi\left(X_{1}, \ldots, X_{P} ; t\right)$ в виде

$$
\psi\left(X_{1}, \ldots, X_{P} ; t\right)=\sum_{\{n\}}(-1)^{\sum_{i<j}\left|n_{i}-n_{j}\right|} \operatorname{det} M,
$$

где суммирование ограничено теми же условиями, что и в (24). Продолжая суммирование в (22), мы получаем неограниченную сумму по всем $n_{1}, \ldots, n_{P}$, что дает нам формулу (7).

Приведенный выше вывод для непрерывного времени легко обобщается на дискретный случай. Комбинаторная трактовка дискретного случая дана в работе [9].

Решение (2)-(6) основного кинетического уравнения (1) дает полное описание полностью несимметричного простого процесса с динамикой исключения для любых начальных условий. Значит, оно может служить отправной точкой для вычисления корреляционных функций многих частиц, а также как основа для более широких прикладных приближений.

\section{3. ОДНОТОЧЕЧНАЯ ВРЕМЕНН АЯ КОРРЕЛЯЦИОННАЯ ФУНКЦИЯ}

Для процесса движения частиц по кольцу с соблюдением правил ASEP в асимптотическом пределе существует стационарное состояние, в котором все конфигурации частиц равновероятны [5]. Пусть по кольцу длины $L$ движутся $P$ частиц, тогда вероятность любой конфигурации равна

$$
P_{\mathrm{st}}=\frac{P !(L-P) !}{L !} .
$$

Теперь, имея решение кинетического уравнения (1), мы можем изучать динамику системы. В частности, мы можем проследить эволюцию системы к стационарному состоянию.

Для примера рассмотрим кольцо длины $L=7$ с тремя частицами. На рис. 2 по оси абсцисс отложено дискретное время эволюции системы $T$, по оси ординат вероятность $P\left(x_{1}, x_{2}, x_{3} ; T \mid x_{1}^{0}, x_{2}^{0}, x_{3}^{0} ; 0\right)$, вычисленная по формулам (2)-(7) для значений $\left\{x_{1}=x_{1}^{0}=1, x_{2}=x_{2}^{0}=2, x_{3}=x_{3}^{0}=3\right\}$. Штриховой линией показано асимптотическое значение вероятности в соответствии с формулой (32).

Зафиксируем начальное положение одной из трех частиц: $x^{0}=1$. Остальные две частицы могут произвольно располагаться на кольце. Вычисляя вероятность того, что через время $T$ какая-либо из частиц снова окажется в точке $x=1$, получим одноточечную временну́ю корреляционную функцию $P(x ; T \mid x ; 0)$. Для $L=7$ эта функция представлена на рис. 3. Штриховой линией показано значение $P=3 / 7$ - вероятность найти какую-либо из трех частиц в узле решетки с координатами $(1, T)$ при $T \rightarrow \infty$. Функция на рис. 3 в колебательном режиме стремится к асимптотическому пределу. Положение экстремумов зависит от средней скорости частиц на кольце, которая в стационарном состоянии имеет точное выражение: $v=(L-P) /(L-1)[5]$. Этот результат есть прямое следствие того факта, что все конфигурации частиц в асимптотическом пределе имеют одинаковую вероятность. 


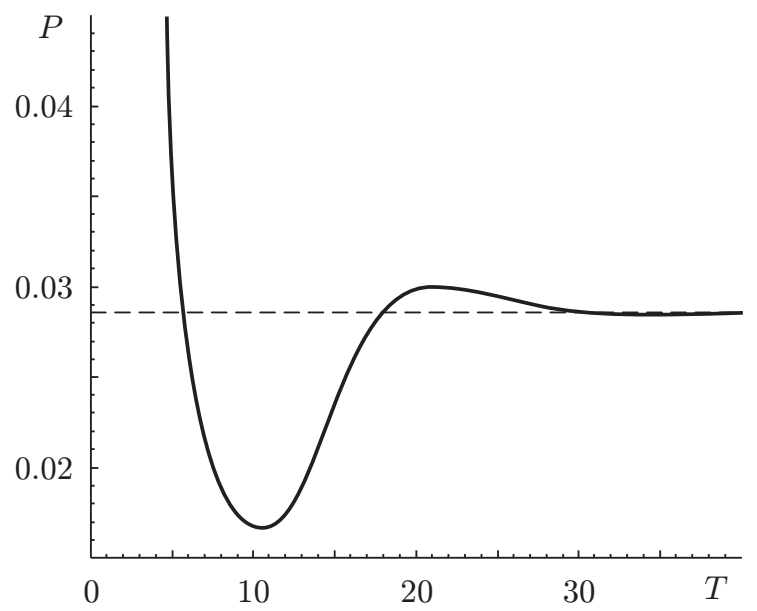

Рис. 2. Условная вероятность $P\left(\alpha ; T \mid \alpha_{0} ; 0\right)$ с течением времени стремится к стационарному значению $P_{\text {st }}$.

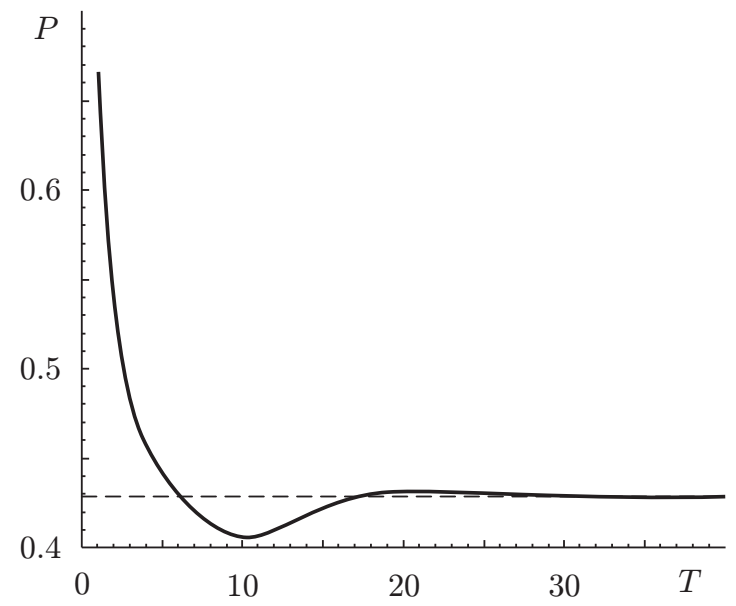

Рис. 3. Одноточечная временна́я корреляционная функция $P(x ; T \mid x ; 0)$ для $L=7, \quad P=3$. 
Все значения функции $P(x ; T \mid x ; 0)$ в интервале $[1,60]$ приведены в таблице.

\begin{tabular}{|r|l|l|l|l|l|l|l|}
\hline 1 & 0.66666667 & 16 & 0.42525535 & 31 & 0.42841195 & 46 & 0.42859134 \\
2 & 0.53020833 & 17 & 0.42750734 & 32 & 0.42836381 & 47 & 0.42858862 \\
3 & 0.47942708 & 18 & 0.42906556 & 33 & 0.42835006 & 48 & 0.42858524 \\
4 & 0.45652669 & 19 & 0.43006409 & 34 & 0.4283606 & 49 & 0.42858173 \\
5 & 0.44108276 & 20 & 0.43061827 & 35 & 0.42838656 & 50 & 0.42857843 \\
6 & 0.42887395 & 21 & 0.43082684 & 36 & 0.42842062 & 51 & 0.42857559 \\
7 & 0.41945044 & 22 & 0.43077798 & 37 & 0.42845712 & 52 & 0.4285733 \\
8 & 0.41242345 & 23 & 0.4305519 & 38 & 0.42849201 & 53 & 0.42857159 \\
9 & 0.40769234 & 24 & 0.43022032 & 39 & 0.42852266 & 54 & 0.42857043 \\
10 & 0.40562123 & 25 & 0.4298446 & 40 & 0.42854769 & 55 & 0.42856974 \\
11 & 0.40639317 & 26 & 0.42947323 & 41 & 0.42856667 & 56 & 0.42856942 \\
12 & 0.40948239 & 27 & 0.42914006 & 42 & 0.42857985 & 57 & 0.42856939 \\
13 & 0.4138095 & 28 & 0.42886449 & 43 & 0.42858795 & 58 & 0.42856956 \\
14 & 0.41828038 & 29 & 0.42865352 & 44 & 0.4285919 & 59 & 0.42856984 \\
15 & 0.42218506 & 30 & 0.42850516 & 45 & 0.42859271 & 60 & 0.42857017 \\
\hline
\end{tabular}

Таким образом, решение основного кинетического уравнения на кольце дает полное описание процесса релаксации системы взаимодействующих частиц из начального состояния в конечное стационарное состояние.

Благодарности. Работа поддержана РФФИ (грант № 03-01-00780-а).

\section{Список литературы}

[1] T.M. Liggett. Interacting Particle Systems. N.Y.: Springer, 1985.

[2] H. Spohn. Large Scale Dynamics of Interacting Particles. N.Y.: Springer, 1991.

[3] D. Dhar. Phase Trans. 1987. V. 9. P. 51.

[4] L.H. Gwa, H. Spohn. Phys. Rev. Lett. 1992. V. 68. P. 725.

[5] B. Derrida. Phys. Rep. 1998. V. 301. P. 65.

[6] S. A. Janovsky, J. L. Lebowitz. Phys. Rev. A. 1992. V. 45. P. 618.

[7] G. M. Schütz. J. Stat. Phys. 1997. V. 88. P. 427.

[8] V. B. Priezzhev. Phys. Rev. Lett. 2003. V. 91. P. 050601.

[9] V.B. Priezzhev. Exact non-stationary probabilities in the asymmetric exclusion process on a ring. cond-mat/0211052. 